\title{
Inhaled Tobramycin in the Treatment of Nosocomial Pneumonia in Severe Sepsis
}

\section{Kuzovlev AN*}

V.A. Negovsky scientific research institute of general reanimatology RAMS, Moscow, Russia

Nosocomial Pneumonia (NP) is the most prevalent intensive care unit infection with the attributable mortality between 5.8 to $27 \%$ [1]. The key etiological agents of NP are associations of multiresistant gram-negative (Pseudomonas aeruginosa, Acinetobacter spp., Klebsiella pneumonia) and gram-positive (Staphylococcus aureus) strains. Rational antibiotic therapy is the background of NP treatment. Intravenous carbapenems, cephalosporins III-IV generations, protected antipseudomonal penicillines, aminoglycosides, fluoroquinolones, glycopeptides and their combinations are recommended for NP treatment. Early start of antibiotics improves outcomes, but the mortality and microbial resistance still remain extremely high. The problem of microbial resistance to the majority of antibiotics is of great significance. Pseudomonas aeruginosa, Acinetobacter spp., Burkholderia spp., Stenotrophomonas spp., have a natural property to form biolayers, which protect them against the immune system and antibiotics. There are currently no perspectives of producing new classes of antibiotics $[2,3]$. In view of the abovementioned special regimens of antibiotic therapy are recommended: increase of doses, continuous infusions, etc. Randomized controlled trial shows that continuous infusion of piperacillin/tazobactam and carbapenems decreases the mortality in NP. The main pitfall of intravenously administered antibiotics is their bad penetration into the lungs, which leads to the sputum concentrations lower than bactericidal. Increasing daily doses of antibiotics is related to a risk of selection of multiresistant strains, sideeffects and superinfection. Therefore Inhaled Antibiotics (IA) as an adjunct to systemic ones presents a good treatment modality $[4,5]$.

\section{Inhaled Antibiotics}

The inhaled root has long been used to administer various medicines: antibiotics, antifungals, antimycobacterials, immune supressors, insulin, vaccines, nitrous oxide, interferones, furosemide, in genotherapy of some diseases. Ehrmann S. et al. showed that $99 \%$ of German doctors use some inhaled preparations, $43 \%$ of them use nebulizers (55\% - jet, $44 \%$ - ultrasound, $14 \%$ - mesh nebulizers). Eighty percent of them use inhaled colistin in their daily practice, and $30 \%$ use inhaled antibiotics minimum 2 times a year [6].

Inhaled colistin, tobramycin, cephalosporins, amphotericin B, pentamydin have been used for prophylaxis and treatment of various infections for more than 50 years now. Modern nebulizers help to administer nearly $50-70 \%$ of IA dose directly into the infection focus. It is noteworthy that in this case the local sputum concentration of antibiotics is significantly higher that after the intravenous administration, which is important when treating multiresistant strains and preventing the formation of resistance. Inhaled administration of antibiotics is related to less systemic toxicity and a profound action on biolayers $[7,8]$.

Inhaled colistin and inhaled aminoglycosides are the most frequently used IA in pulmonology and intensive care medicine $[7,8]$. Aminoglycosides are the most suitable antibiotics for inhalation because they are bactericidial and concentration-dependant. Also inhaled fluoroquinolones, cephalosporines, liposomal aminoglycosides; aztreonam, combinations (fosfomycin/tobramycin, colitin/tobramycin, ciprofloxacin/colistin) are used. Inhaled fosfomycin is active against both gram-negatives and gram-positives, but it is strongly recommended to combine it with other antibiotics to prevent a rapid resistance formation [9]. It is inexpedient to use inhaled beta-lactames, because they are concentration-dependant antibiotics, and therefore multiple inhalations will be required (e.g. every $3 \mathrm{hrs}$ for ceftazidime). Carbapenems when inhaled induce allergic reaction [10].

The majority of IA are used to treat acute and chronic pseudomonal infection in cystic fibrosis and bronchoectatic disease. Inhaled tobramycin (IT), colistin (50-75 mg BID-TID), aztreonam (75 mg TID within 28 days) and other antibiotics are used for continuous treatment of infectious complications of cystic fibrosis both in- and out-hospital. A 28-day course of IT is proved to be effective in eradication of Pseudomonas aeruginosa in cystic fibrosis $(300 \mathrm{mg} /$ day within 28 days, then 28 -days break). But the recent meta-analysis shows that there are currently no evident data to support IA use in cystic fibrosis. Moreover, the increase of the prevalence of colistin and aminoglycoside resistant strains of Pseudomonas aeruginosa and gram-positive microbes is detected in cystic fibrosis patients $[11,12]$.

There were no randomized multicenter trials of IA use in NP. Several small trials proved that IA in combination with systemic antibiotics decrease the symptoms of NP, facilitate weaning from ventilator, decrease the sputum microbes titer. There are also some data on IA efficacy in nosocomial tracheobronchitis. Lu et al. [13] showed the same efficacy of systemic and inhaled ceftazidime and amikacin, but a lower rate of resistance formation in IA groups. It is noteworthy that IA in this study were used as a monotherapy (ceftazidime $15 \mathrm{mg} /$ $\mathrm{kg}$ every $3 \mathrm{hrs}$. amikacin $25 \mathrm{mg} / \mathrm{kg} /$ day). Several cases of exhalation filter obstruction were detected [13]. The same group of authors proved later the same efficacy of inhaled colistin and combination of intravenous beta-lactames and aminoglycosides in NP patients caused by Pseudomonas aeruginosa and Acinetobacter baumanii [14]. Korbila et al. [15] showed more rapid NP resolution in combination of inhaled and intravenous forms of colistin [15]. Arnold et al. [16] in the retrospective trial showed a higher survival in NP patients treated with IT [16]. All the above mentioned trials showed a low threshold of resistance emergence and low incidence of side effects in IA use.

Our data on the inhaled tobramycin (Bramitob, Chiesi Farmaceutici, Italy) use in septic patients with NP proved its efficacy and safety: decrease of systemic inflammation and acute respiratory insufficiency signs $2.3 \pm 1.2$ after the treatment onset. Eradication of microbes in sputum was detected in $28 \%$ of patients; in other patients a decrease of microbial titer to $10^{3-4} \mathrm{CFU} / \mathrm{ml}$ was detected. Deescalation

${ }^{*}$ Corresponding author: Kuzovlev AN, V.A. Negovsky scientific research institute of general reanimatology RAMS, Moscow, Russia, Tel: 892-6188-7641; E-mail: artem_kuzovlev@mail.ru

Received December 10, 2013; Accepted December 12, 2013; Published December 14, 2013

Citation: Kuzovlev AN (2013) Inhaled Tobramycin in the Treatment of Nosocomial Pneumonia in Severe Sepsis. J Pulm Respir Med 4: e132. doi:10.4172/2161-105X $1000 \mathrm{e} 132$

Copyright: $\odot 2013$ Kuzovlev AN. This is an open-access article distributed under the terms of the Creative Commons Attribution License, which permits unrestricted use, distribution, and reproduction in any medium, provided the original author and source are credited. 
of antibiotic therapy was possible in $20 \%$ of patients treated with IT. It is noteworthy that $20 \%$ of patients were in vitro resistant to tobramycin, but it was clinically effective, probably due to a local superconcentration. Treatment with IT was associated with an increase of sensitivity of microbes to antibiotics they were prior resistant to (40\% of patients). This is probably due to IT effects on biolayers. Positive chest X-ray dynamics was detected in $60 \%$ of patients $9.0 \pm 2.5$ days after the treatment onset. The treatment with IT made it possible to wean $30 \%$ of patients on the day $5.2 \pm 1.7$. Hearing loss and tinnitus was detected only in 2 patients in our study. There were no cases of bronchospasm or kidney dysfunction in our study, which is in accordance with the other trials $[17,18]$.

Only special preparations for inhalation and modern nebulizers must be used for an effective treatment with IA. The preparation for inhalation use should not contain some conservatives and should not be hyperosmolar, should be $\mathrm{pH}$ neutral and contain chlorides to prevent bronchospasm and cough. Mesh nebulizers are most suitable for IA administration. This type of nebulizers forms $2.1 \mu \mathrm{m}$ particles and provides a delivery of 5-70\% of drug dose into the lungs; temperature of preparation remains constant during the aerosol formation; the air flow minimally affects the ventilation parameters; constant humidification of air can be continued. Instillation of antibiotics through the intubation or tracheostomic tube is ineffective and must never be used [19].

Inhaled antibiotics are not used as a monotherapy without the systemic antibiotics, because their absorbtion into the blood is low (2-4\%) and not sufficient to treat the concomitant extrapulmonary infections and moreover insufficient to reach the alveoli [19]. But we have a clinical experience of an effective monotherapy with IT in a patient with severe allergic reaction to systemic antibiotics. Currently it is not recommended to use IA for the NP prophylaxis [15-19].

\section{Use of IA is related to some problems}

The penetration of IA into the obstructed airways is deteriorated. A possible inactivation of IA in sputum should be taken into account. This effect is mostly profound in aminoglycosides. A 25 -fold increase over the minimal inhibitory concentration is required to overcome this inactivation. Changes of physico-chemical properties of IA during the aerosol formation due to heating, cooling, vibration, etc. (more profound in jet nebulizers), local and systemic toxic effects, bronchoconstrictive effects of conservatives should be noted. The bronchospasm is mostly induced by the inhaled colistin. Only special preparations for inhalation must be used to prevent these complications. Inhaled antibiotics and nebulizers are expensive, their use is associated with the environment pollution and resistance formation [17-19].

Thus, the use of IA in combination with the intravenous ones is an efficient and safe treatment modality for severe NP caused by gramnegative strains. In spite of low evidence for this treatment method, the current situation of high microbial resistance and no perspectives of new antibiotics development raise the significance of this treatment modality.

\section{References}

1. Bekaert M, Timsit JF, Vansteelandt S, Depuydt P, Vésin A et al (2011) Outcomerea Study Group. Attributable mortality of ventilator-associated pneumonia: A reappraisal using causal analysis. Am J Respir Crit Care Med 184: 1133-1139.

2. Goloubev AM, Smelaya TV, Moroz VV, Popov AA, Tolbatov AA (2010) Outof-hospital and nosocomial pneumonia: clinical and morphological properties. General reanimatology VI: 5-14

3. Gilbert DN, Moellering RC, Eliopoulos GM, Chambers HF, Saag MS (2012) The Sanford guide to antimicrobial therapy 2012. Antimicrobial therapy Inc.
4. Tamma PD, Putcha N, Suh YD, Van Arendonk KJ, Rinke ML (2011) Does prolonged $\beta$-lactam infusions improve clinical outcomes compared to intermittent infusions? A meta-analysis and systematic review of randomized, controlled trials. BMC Infect Dis 11: 181.

5. Falagas ME, Tansarli GS, Ikawa K, Vardakas KZ (2013) Clinical outcomes with extended or continuous versus short-term intravenous infusion of carbapenems and piperacillin/tazobactam: a systematic review and meta-analysis. Clin Infect Dis 56: $272-282$

6. Ehrmann S, Roche-Campo F, Sferrazza Papa GF, Isabey D, Brochard L, et al. (2013) Aerosol therapy during mechanical ventilation: an international survey. Intensive Care Med 39: 1048-1056

7. Dhand $R$ (2007) The role of aerosolized antimicrobials in the treatment of ventilator-associated pneumonia. Respir Care 52: 866-884.

8. Ioannidou E, Siempos II, Falagas ME (2007) Administration of antimicrobials via the respiratory tract for the treatment of patients with nosocomial pneumonia: a meta-analysis. J Antimicrob Chemother 60: 1216-1226.

9. MacLeod DL, Barker LM, Sutherland JL, Moss SC, Gurgel JL, et al. (2009) Antibacterial activities of a fosfomycin/tobramycin combination: a novel inhaled antibiotic for bronchiectasis. J Antimicrob Chemother 64: 829-836.

10. Hilas O, Ezzo DC, Jodlowski TZ (2008) Doripenem (doribax), a new carbapenem antibacterial agent. P T 33: 134-180.

11. Chuchalin A, Amelina E, Bianco F (2009) Tobramycin for inhalation in cystic fibrosis: Beyond respiratory improvements. Pulm Pharmacol Ther 22: 526-532.

12. Drobnic ME, Suñé $P$, Montoro JB, Ferrer A, Orriols R (2005) Inhaled tobramycin in non-cystic fibrosis patients with bronchiectasis and chronic bronchial infection with Pseudomonas aeruginosa. Ann Pharmacother 39: 39-44.

13. Lu Q, Yang J, Liu Z, Gutierrez C, Aymard G, et al. (2011) Nebulized ceftazidime and amikacin in ventilator-associated pneumonia caused by Pseudomonas aeruginosa. Am J Respir Crit Care Med 184: 106-115.

14. Lu Q, Luo R, Bodin L, Yang J, Zahr N, et al. (2012) Efficacy of high-dose nebulized colistin in ventilator-associated pneumonia caused by multidrugresistant Pseudomonas aeruginosa and Acinetobacter baumannii. Anesthesiology 117: 1335-1347.

15. Korbila IP, Michalopoulos A, Rafailidis PI, Nikita D, Samonis G, et al. (2010) Inhaled colistin as adjunctive therapy to intravenous colistin for the treatment of microbiologically documented ventilator-associated pneumonia: a comparative cohort study. Clin Microbiol Infect 16: 1230-1236.

16. Arnold HM, Sawyer AM, Kollef MH (2012) Use of adjunctive aerosolized antimicrobial therapy in the treatment of Pseudomonas aeruginosa and Acinetobacter baumannii ventilator-associated pneumonia. Respir Care 57 1226-1233.

17. Moroz VV, Kuzovlev AN, Polovnkov SG, Stec VV, Varvarin VV (2012) Inhaled tobramycin in treatment of severe nosocomial pneumonias. General reanimatology VIII: 5-10.

18. Polovnikov S, Kuzovlev A, Iljichev A (2011) Administration of inhaled tobramycin in severe nosocomial pneumonia (case report). Pulmonology 2: 109-112.

19. Le J, Ashley ED, Neuhauser MM, Brown J, Gentry C, et al. (2010) Consensus summary of aerosolized antimicrobial agents: application of guideline criteria Insights from the Society of Infectious Diseases Pharmacists. Pharmacotherapy 30: $562-584$ 